\title{
FEATURES OF THE SOURCE TERM CALCULATION CAPABILITY OF THE NECP-X CODE
}

\author{
Xingjian Wen ${ }^{1}, Z^{2 h o u y u ~ L i u}{ }^{1}$, Kai Huang ${ }^{2}$, Liangzhi Cao $^{1}$ \\ 1: School of Nuclear Science and Technology, Xi'an Jiaotong University, \\ 28 West Xianning Road, Xi'an, Shaanxi 710049, China \\ 2: Institute of Applied Physics and Computational Mathematics, People's Republic of China \\ *zhouyuliu@xjtu.edu.cn
}

\begin{abstract}
The source term calculation capability is developed for the high-fidelity neutronics code NECP-X. Generally, a full activation library is used, but the memory requirement is unacceptable for the high-fidelity calculation. In order to minimize the memory requirement during the calculation with very strict conditions, a new generalized activation chain compressed method is proposed based on the influence qualification. One basic compression element is a reaction channel or an isotope, and the influence of every compression element to the final results are qualified. To enlarge the range of application of the new compressed library, an effective method to determine representative problems, which utilizes the neutron spectra and neutron flux, is developed and analyzed. Based on the ENDF-VII.0, EAF-2010 evaluated nuclear library and the influence qualification-based activation library compression method, a new compressed activation library is generated. The VERA-3A problem and the KAIST problem are used to assess the accuracy and the efficiency of the new activation library. 85 measurements of decay heat from decay heat measurement facilities GE-Morris and CLAB are used to validate the decay heat calculation in NECP-X. The results show good accuracy of NECP-X in predicting radiation source term of the spent nuclear fuel and significant memory saving when using new compressed activation library.
\end{abstract}

KEYWORDS: NECP-X, activation chain compression, source term, decay heat validation

\section{INTRODUCTION}

Radiation source term is a very important parameter for radiation shielding design for nuclear facilities such as fuel storage pools, Spent Nuclear Fuel (SNF) transport systems, spent-fuel interim storage facilities and spent-fuel reprocessing plants. Accurate prediction of the source term could support making full use of these facilities and improve the economic efficiency.

The traditional two-step method pays more attention to reactivity and power distribution of the reactor, and it could not explicitly track every nuclide in fuel stacks and structure materials. Normally, ORIGENARP is adopted to conduct the source term analysis with extremely conservative operation conditions, which means the result is of great margin. With the development of the computers, the whole-core calculations now can be performed by the one-step calculation, and pretty precise results can be obtained by using the detailed transmutation chain and geometry description, including the pin-wise pin power distribution, accurate reactivity and sub-pin-wise nuclide inventory. The appearance of the one-step 
whole-core high-fidelity calculation method provides the potential to calculate the radiation source term of SNF after the reactor shutdown accurately.

In order to reduce the computation burden, compressed depletion chain has been widely used for the depletion calculation in fuel stacks. However, not much attention was paid to the compression of activation chain for the activation calculations of structure materials because of the conservative calculation strategy. Estimated memory for the high-fidelity calculation indicate that neutronics and depletion calculation (compressed depletion chain is adopted) take up about $1.8 \mathrm{~TB}$ and $0.3 \mathrm{~TB}$, respectively. However, at least $6 \mathrm{~TB}$ of memory will be needed for the calculation of activation photon intensity and 18-group spectrum. A generalized activation chain compressed method is not found in the publications, so it would be significant to develop a new generalized activation chain compressed method.

NECP-X is a three-dimensional (3-D) whole core reactor physics code being developed at Xi'an Jiaotong University for high-fidelity calculation of the numerical reactor since 2015. NECP-X is able to carry out steady-state calculation, multi-cycle calculation and transient calculation, which has been widely verified and validated [1-5]. In this work, a new generalized activation chain compression method is proposed and the source term capability of NECP-X is developed. The content is organized as follows. In section 2, the overview of NECP-X is described, including neutronics models and the depletion models. Next a generalized activation chain compression method based on the importance qualification analysis is presented in detailed. Theory of the source term calculation implemented in NEPC-X are shown in section 4. In section 5, efficiency and accuracy of the new compressed activation library is evaluated. The assessment of calculation models to predict the decay heat, verification and validation results of source term calculation are shown in section 6. Conclusions are summarized in section 7.

\section{A GENERALIZED ACTIVATION CHAIN COMPRESSED METHOD BASED ON INFLUENCE QUALIFICATION}

\subsection{Analysis on the features of Bateman equations in the activation calculation}

The accuracy of solving Bateman equations depends on the microscopic reaction rates of neutron-induced reactions and decay constants of decay reactions. In the activation calculation, the multi-group activation cross sections are constants for every reaction channel, thus the neutron spectrum and the level of singlegroup neutron flux dominate the solutions Bateman equations.

On the one hand, if the neutron spectrum is assumed to be constant, the microscopic reaction rates have a positive correlation with the neutron flux. On the other hand, with the assumption of constant neutron flux, the neutron spectrum is the only factor having impact on microscopic reaction rates. In the activation calculation, two main neutron-induced reactions are the radiation capture reactions and the threshold reactions, such as the $(\mathrm{n}, 2 \mathrm{n})$ reaction. For radiation capture reactions, the cross sections obey $1 / \mathrm{v}$ law in general, which means harder neutron spectrum may produce smaller microscopic reaction rates and softer neutron spectrum will make microscopic reaction rates higher. In contrast to the threshold reactions, harder neutron flux spectrum may generate bigger microscopic reaction rates and softer neutron flux spectrum will make microscopic reaction rates smaller.

\subsection{Influence qualification of the basic compression element}

To analyze all possible transmutation paths in the full-fidelity activation chain, all the transfer relations are defined as the basic compression elements. According to their characteristics, they can be classified into two categories: the neutron-induced reaction channel and the decay path. For each activation regions of the specific problem, the activation photon spectrum at each decay step is expressed as follows: 


$$
P h o_{i g, \text { ireg }, t}=\sum_{\text {inuc }=1}^{n n u c} P h o_{\text {inuc, }, \text { ig, }, \text { reg }, t}
$$

Where $P h o_{i g, t}$ is the photon spectrum of photon energy group ig at decay time $t$ in the activation region ireg, ${ }^{P h o_{\text {inuc, }, \text { g, ireg, } t}}$ is the photon spectrum of photon emitter inuc, nnuc is the total number of isotopes in the activation chain.

The influence of one basic compression element can be defined as the accuracy loss of the activation photon spectrum from deleting this basic compression element:

$$
L(\text { ig }, \text { ireg }, t)_{\text {photon }}=\frac{\left|P h o_{i g, \text { ireg }, t}^{\text {new }}-P h o_{\text {ig, }, \text { ireg }, t}\right|}{P h o_{\text {ig, ireg }, t}}
$$

Where $P h o_{i g, i r g, t}$ is the photon spectrum based on the full-fidelity activation chain, $P h o_{i g, \text { ireg }, t}^{\text {new }}$ is the photon spectrum based on the activation chain after deleting one specific basic compression element, $L(i g, \text { ireg, } t)_{\text {photon }}$ is the relative difference.

Finally, the significance of the basic compression element can be calculated as:

$$
s=\max _{\text {ireg } \in \mathbf{R}, i g \in \mathbf{G}, t \in \mathbf{T}}\left(L(\text { ig }, \text { ireg }, t)_{\text {photon }}\right)
$$

Where $\mathbf{R}, \mathbf{G}, \mathbf{T}$ represent the set of representative problems, energy groups of activated photon spectra and decay time, respectively.

The determination of the activation photon source term is based on the radioactivity of activation products and their decay products. Therefore, when the activation photon spectrum is utilized to assess the influence of the basic compression element, the accuracy of the radioactivity and decay heat can also be considered.

\subsection{Generalization of the new compressed method}

The neutron spectrum and the level of the single-group flux are different for the structure materials in the nuclear power plant, and it is difficult to use one particular neutron spectrum and flux for the compression of the activation chain. Thus, a set of representative problems need to be determined to qualify the influence of every compression element.

Firstly, the influences of one basic compression element with different calculation conditions are different and there is a variation range of influence for every basic compression element. Then the maximal point and minimal point of the basic compression element are defined, which means the maximum value and minimum value of the influence of the basic compression element. Fig. 1 has shown the relationship between extreme values of influence and the compressed criteria, which can be analyzed as following.

If maximal point of one specific basic compression element is smaller than the compressed criterion, which means that the influence of this basic compression element is smaller than the compressed criterion in any cases and it can be deleted.

1) If minimal point is larger than the compressed criterion, the basic compression element is significant for activation photon spectrum calculation and it will be saved during the simplification of the 
activation chain.

2) If the compressed criterion located between maximal point and minimal point, saving this basic compression element is conservative.

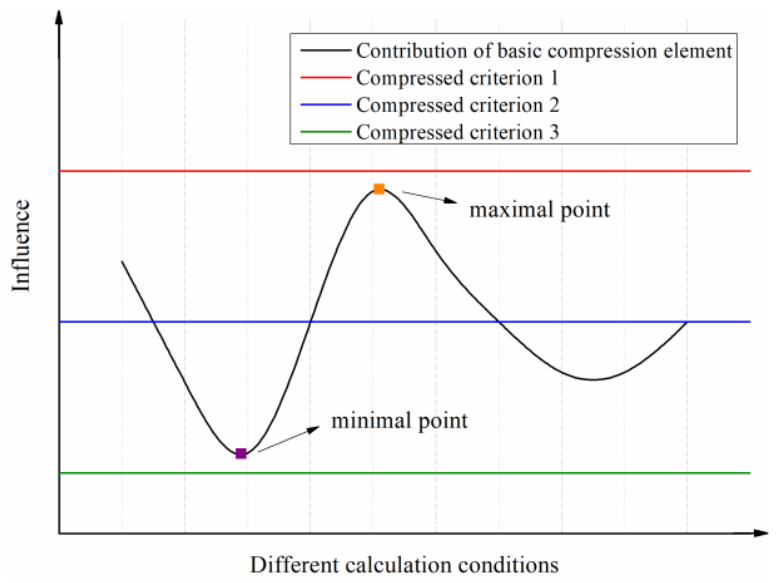

Figure 1. The relationship between extreme values of influence and the compressed criteria

If the microscopic reaction rate of a neutron-induced reaction is large enough, this neutron-induced reaction channel is important and all decay reactions are relatively unimportant. In reverse, small microscopic reaction rates make neutron-induced reaction channels less important and the decay reactions more important. The influences of neutron-induced reactions have positive correlation with their microscopic reaction rates. In reverse, smaller microscopic reaction rates make neutron-induced reaction channels less important and the influences of decay reactions will be larger.

As analyzed in the last part, extreme neutron fluxes and neutron spectra can generate highest and lowest microscopic reaction rates in the activation calculation. Based on discussion above, the neutron spectrum and the single-group flux used for compression are specified for each structure material. For the neutron spectrum, hardest neutron spectrum and softest spectrum of the whole life of the reactor are considered. As for the level of the single-group flux, the highest and lowest level of fluxes are adopted for the compression of the activation chain.

\section{GENERATION OF THE NEW COMPRESSED ACTIVATION LIBRARY}

\subsection{Flowchart of the generation of activation library}

The overall flow of the generation of full-fidelity activation library and the activation compression method is illustrated in Fig. 2. During the procedure of the generation of the full-fidelity activation library, several sub-libraries of ENDF/B-VII.0 library and EAF-2010 are integrated and reformatted to the customized format. The full-fidelity activation library can guarantee an accuracy source term calculation with large quantities of isotopes and reaction channels. Based on the full-fidelity activation library, the newly proposed compression method is used to reduce the scale of the activation library. Firstly, some useless isotopes, which will not appear in the reactor, are deleted. Then a set of representative problems are used for the evaluation of the influences of basic compression element. Then the compression element with low influences are removed (in this work, compression elements whose influences are less than $0.1 \%$ will be deleted). Finally, isolated isotopes, whose transmutation paths are all deleted, will be eliminated. The new compressed activation library is supposed to significantly reduce the memory requirement for the activation photon spectra calculation. 


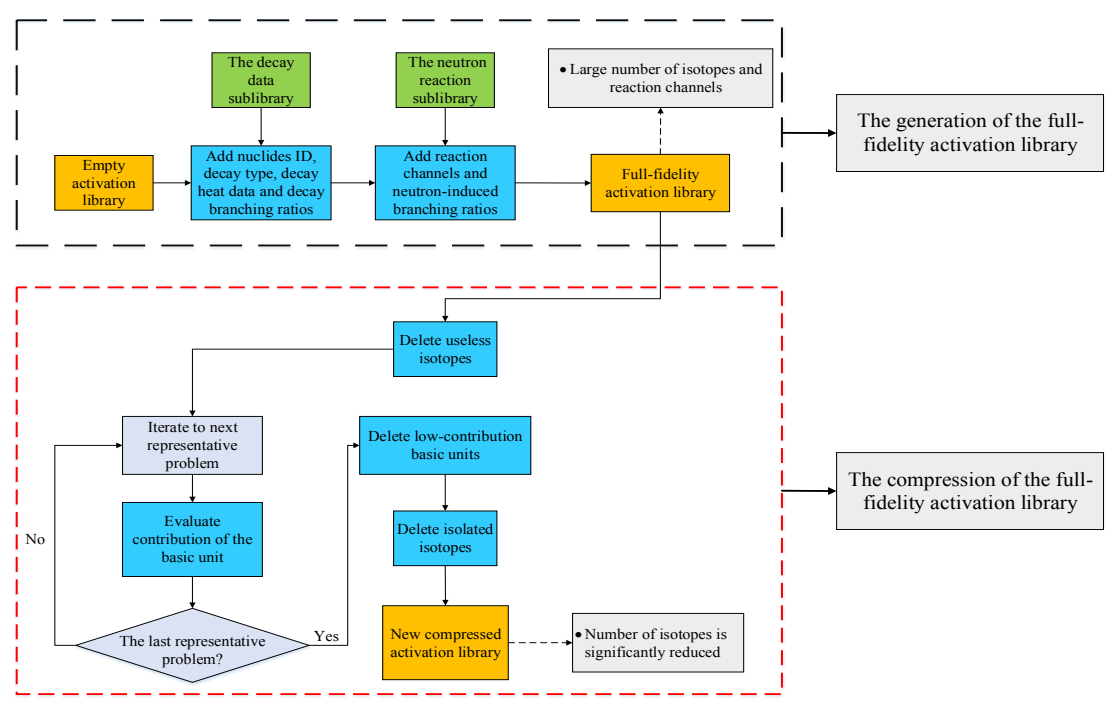

Figure 2. Flowchart of the NECP-X activation library generation and compression system

\subsection{Specification of the new compressed activation library}

The compression was performed with seven cores of the 2.6-GHz Intel Xenon Gold 6132 and the total time was about $1.5 \mathrm{~h}$. The comparison of the full-fidelity activation library and the new compressed activation library is presented in Table II. The number of nuclides tracked during the calculation is reduced from 3838 to 353 and the number of transmutation paths also has been significantly decreased.

Table II. Main characteristics of the full-fidelity library and new compressed library

\begin{tabular}{|c|c|c|c|c|c|c|}
\hline Library & description & $\begin{array}{c}\text { \# of } \\
\text { nuclides }\end{array}$ & $\begin{array}{c}\text { \# of } \\
\text { decay } \\
\text { paths }\end{array}$ & $\begin{array}{c}\text { \# of } \\
\text { decay } \\
\text { daughters }\end{array}$ & $\begin{array}{c}\text { \# of neutron- } \\
\text { induced paths }\end{array}$ & $\begin{array}{c}\text { \# of neutron- } \\
\text { induced } \\
\text { daughters }\end{array}$ \\
\hline $\begin{array}{c}\text { actlib_383 } \\
8\end{array}$ & Full-fidelity library & 3838 & 3748 & 5967 & 9002 & 9250 \\
\hline actlib_353 & Compressed library & 353 & 236 & 208 & 3617 & 489 \\
\hline
\end{tabular}

\section{SOURCE TERM CAPABILITY IN NECP-X}

Radiation source terms considered in NECP-X consist of several elements: the activity and decay heat of the whole spent system, neutron source and the activation photon source. The activity and decay heat have multiple origins, including the decay of actinides and fission products of fuel stacks and activation products of structure materials. The neutron source of SNF consists of neutrons coming from the (alpha, $n$ ) reactions and the spontaneous fission in fuel stacks. As for the activation photon source term, gamma along with the produce of the decay of the activation products is the main source. The methodology of source term calculation in NECP-X is similar with those of SCALE6.1 and detailed theory is not presented here because of limited space of paper. 


\section{NUMERICAL RESULTS}

\subsection{Efficiency and accuracy of the new compressed activation library}

The fuel assembly VERA-3A in the WBN1 reactor [6] and the KAIST small PWR core [7] are used to verify the new compressed activation library. The specification of VERA-3A and KAIST core are presented in Figure 3, which are originally steady-state cases. In this work, depletion and activation calculation is implemented. The power density for this case is $40 \mathrm{MW} / \mathrm{tU}$. The final irradiation time for VERA-3A and KAIST core are 1500 EFPD and 500EFPD, respectively. When the irradiation calculation is finished, a 30-year decay calculation is followed. In the calculation, all fuel regions are identified as the depletion regions and independent activation calculation is implemented for all structure materials. 3528 depletion regions and 6818 activation regions are considered in the calculation of VERA-3A. As for KAIST core, 3168 depletion regions and 4104 activation regions are included in the calculation.

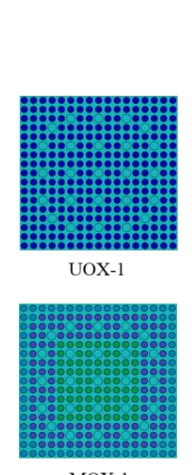

MOX-1

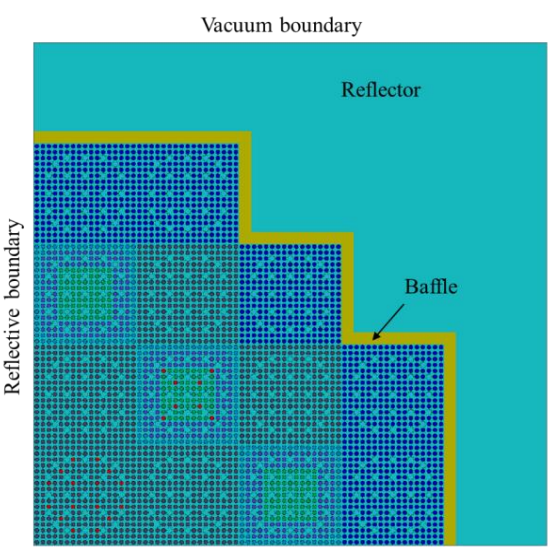

Reflective boundary

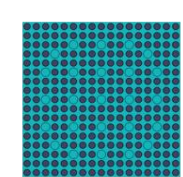

UOX-2
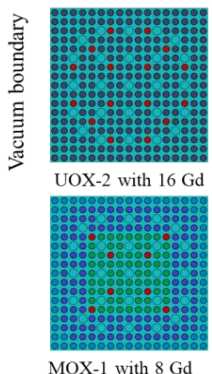

MOX-1 with $8 \mathrm{Gd}$
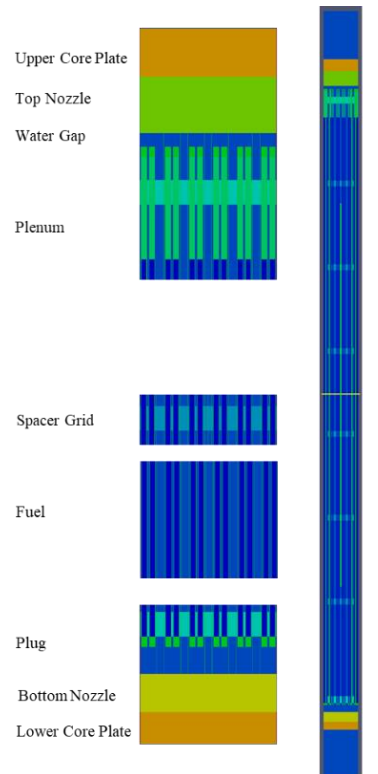
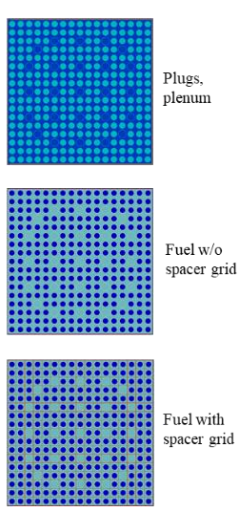

Figure 3. Specification of the KAIST core and VERA-3A assembly
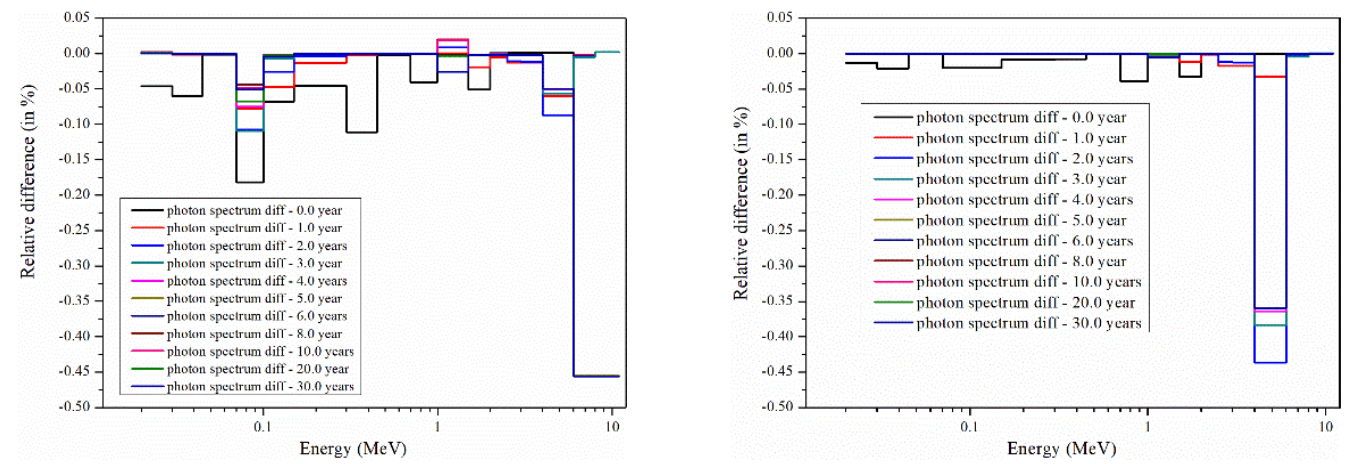

Figure 4. Relative difference of photon spectra of the VERA-3A and KAIST core 
The results have concluded in Figure 4, which indicates that the maximum relative difference of photon spectra of all decay time is $-0.46 \%$ and $-0.36 \%$ for VERA-3A and KAIST core, respectively. As for the storage consumption of activation photon spectra calculation, the memory is estimated as $24.8 \mathrm{~GB}$ and $14.93 \mathrm{~GB}$ using the full-fidelity activation library actlib_3838 to $2.54 \mathrm{~GB}$ and $1.53 \mathrm{~GB}$ using the new compressed activation library actlib_353 for VERA-3A and KAIST core.

\subsection{Comparison to the decay heat measurements}

Decay heat measurements of 48 spent full-length fuel assemblies are used for the validation of NECP-X. The measurements are performed in two separate facilities in the United State and Sweden, including the General Electric Morris Operations (GE-Morris) spent fuel storage facility in Morris and the Swedish Central Interim Storage Facility for Spent Fuel (CLAB) in Oskarshamn. A summary of the experimental data used is provided in Table. III, which contains the measurement facility, the reactor name, the assembly design, the enrichment of the fuel pellets, number of measurements and the average burnup of assemblies. The fuel enrichment is from $2.100 \mathrm{wt}$ \% $235 \mathrm{U}$ to $4.005 \mathrm{wt} . \% 235 \mathrm{U}$, and the average burnup of assemblies is from $32.4 \mathrm{GWd} / \mathrm{tU}$ to $50.9 \mathrm{GWd} / \mathrm{tU}$.

Table III. Summary of full-length fuel assembly measurements

\begin{tabular}{|c|c|c|c|c|c|c|}
\hline $\begin{array}{c}\text { Measurement } \\
\text { Facility }\end{array}$ & Reactor & $\begin{array}{c}\text { Assembly } \\
\text { design }\end{array}$ & $\begin{array}{c}\text { Enrichment } \\
\text { (wt.\% U235) }\end{array}$ & $\begin{array}{c}\text { No. of } \\
\text { measurements } \\
\text { / assemblies }\end{array}$ & $\begin{array}{c}\text { Cooling } \\
\text { time (day) }\end{array}$ & $\begin{array}{c}\text { Average Burnup } \\
\text { (GWd/tU) }\end{array}$ \\
\hline \multirow{2}{*}{ GE-Morris } & $\begin{array}{c}\text { Point Beach } \\
2\end{array}$ & $14 \times 14$ & 3.397 & $6 / 6$ & $1629-1635$ & 39.4 \\
\cline { 2 - 7 } & San Onofre 1 & $14 \times 14$ & $3.865-4.005$ & $8 / 8$ & $1078-3012$ & 32.4 \\
\hline \multirow{2}{*}{ CLAB } & Ringhals 2 & $15 \times 15$ & $3.095-3.252$ & $33 / 18$ & $6198-8468$ & 50.9 \\
\cline { 2 - 7 } & Ringhals 3 & $17 \times 17$ & $2.100-3.404$ & $38 / 16$ & $5818-7304$ & 47.3 \\
\hline
\end{tabular}

Comparisons of the calculated value and measurements from GE-Morris and CLAB are presented in Table IV. As shown in the Table IV, the results of NECP-X for all measurements agree well with the measured data. The maximum value of the mean values and the standard deviations is $-0.58 \%$ and $1.41 \%$, respectively.

Table IV. Summary of average C/E-1 (\%) for GE-Morris and CLAB measurements

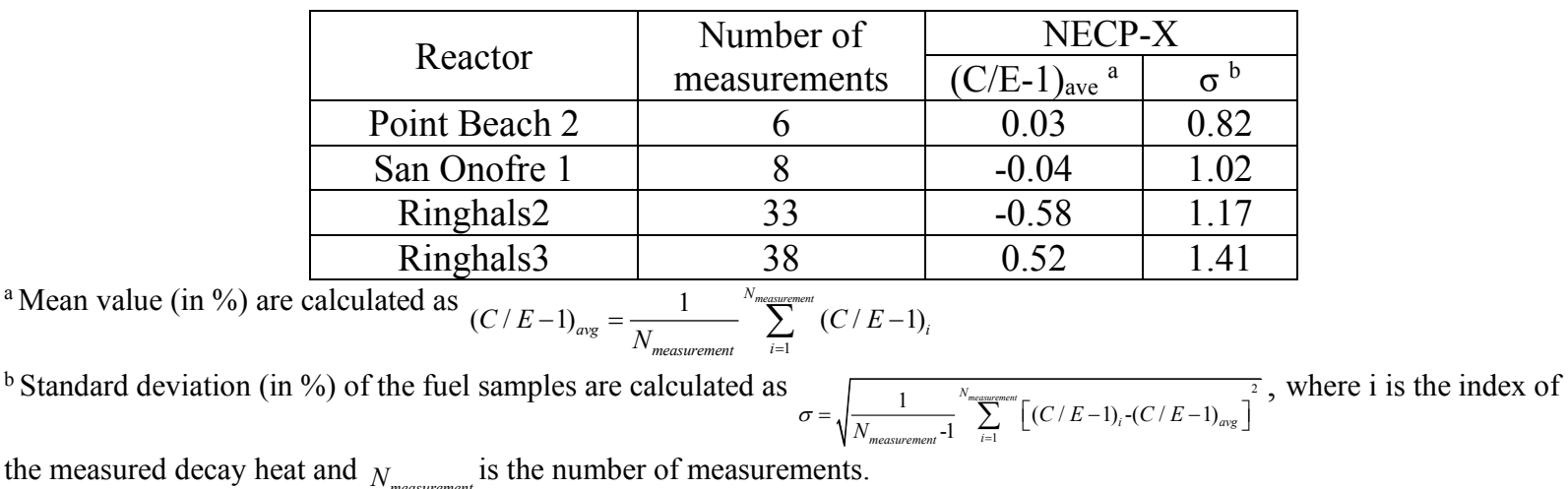




\section{CONCLUSIONS}

A new generalized activation chain compressed method to minimize the size of activation library, which will reduce the computational burden and memory requirement, is proposed. The new compressed method, including how to select the representative problems, are presented and discussed in detailed. Based on the ENDF-VII.0 and EAF-2010 evaluated nuclear library, the full-fidelity activation library actlib_3838 and a new compressed activation library actlib_353 is generated. The methodology of source term calculation is implemented in the NECP-X code. The VERA-3A problem and the KAIST problem are utilized to assess the accuracy and the efficiency of the new activation library. The results demonstrate that the new compressed activation library could keep good accuracy and achieve significant memory saving. The maximum accuracy loss of using the compressed library is less than $0.5 \%$ and the memory is reduced by approximately a factor of 10.85 measurements of decay heat from GE-Morris and CLAB are used to validate the decay heat calculation of NECP-X. The validation results of source term calculation show good accuracy of NECP-X in predicting decay heat of the spent nuclear fuel.

\section{ACKNOWLEDGMENTS}

This work was supported by the National Natural Science Foundation of China [grant number 11605128], [grant number 11735011].

\section{REFERENCES}

1. Chen, J., et al., A new high-fidelity neutronics code NECP-X. Annals of Nuclear Energy, 2018. 116: p. 417-428.

2. Liu, Z., et al., Development and verification of the high-fidelity neutronics and thermal-hydraulic coupling code system NECP-X/SUBSC. Progress in Nuclear Energy, 2018. 103: p. 114-125.

3. Liu, Z., et al., Improvement and optimization of the pseudo-resonant-nuclide subgroup method in $N E C P-X$. Progress in Nuclear Energy, 2018. 103: p. 60-73.

4. Liu, Z., et al., The pseudo-resonant-nuclide subgroup method based global-local self-shielding calculation scheme. Journal of Nuclear Science and Technology, 2018. 55(2): p. 217-228.

5. Zhao, C., et al., Improved leakage splitting method for the 2D/1D transport calculation. Progress in Nuclear Energy, 2018. 105: p. 202-210.

6. Godfrey, A., VERA Core Physics Benchmark Progression Problem Specifications. Revision 4, CASL Technical Report: CASL-U-2012-0131-002. 2014.

7. Cho, N.Z., Benchmark Problems in Reactor and Particle Transport Physics. 2000, Korea Advanced Institute of Science and Technology Department of Nuclear and Quantum Engineering Nuclear Reactor Analysis and Particle Transport Lab 